\title{
Commentary
}

\section{Leveraging Community Engaged Policy, Systems, and Environmental (PSE) Approaches to Foster Healthy Eating in the United States}

\author{
Amy L. Yaroch ${ }^{1}$, Courtney A. Parks ${ }^{1}$, and Mary T. Story ${ }^{2}$ \\ ${ }^{1}$ Gretchen Swanson Center for Nutrition, Omaha, Nebraska \\ ${ }^{2}$ Duke Global Health Institute, Duke University; \\ Healthy Eating Research National Program, Robert Wood Johnson Foundation
}

Poor dietary quality in the United States (U.S.) continues to be a major public health concern, with approximately threefourths of Americans reporting an eating pattern that is low in fruits, vegetables, and whole grains, and that is high in added sugars, saturated fats, sodium, and calories (U.S. Department of Health and Human Services \& U.S.

Department of Agriculture, 2015). Consequently, poor dietary quality contributes to the burden of chronic diseases (e.g., obesity, type 2 diabetes, cardiovascular disease, some types of cancer). Differences in dietary quality among socioeconomic subgroups also contributes to disparities, with low-income and racial/ethnic minority populations experiencing higher rates of chronic diseases and food insecurity. Unfortunately, these disparities have existed for a long time and are being further exasperated by the COVID-19 global pandemic. Obesity, which is at an alltime high in the United States, has emerged as a major risk factor for hospitalization, intensive care, and mortality among individuals with COVID-19 infections (Centers for Disease Control and Prevention, n.d.).

Long before the pandemic, structural and systemic factors in our society were contributing to deep inequities in access to and availability of healthy and affordable foods for many Americans. The COVID-19 pandemic has exposed and exacerbated food insecurity and the weaknesses in our U.S. food system and the long-standing disparities among low-income households and people of color (Shanks et al., 2020). Due to unprecedented job loss and economic downturn during the pandemic, rates of food insecurity have spiked upwards of $28 \%$ of households (and $42 \%$ of those with children) (Schanzenbach \& Pitts, 2020).

Food security in the U.S. is typically defined as "access by all people at all times to enough food for an active, healthy life." However, the Food Agriculture Organization (FAO) expands upon this by describing the quality of food, endorsing individual needs and preferences, and sustainability over time. One area in need of bolstering is measurement of food security. Currently in the U.S., food security is based upon a core set of metrics focused on economic resources to purchase food, but moving forward it should also be expanded to monitor and address variables that are associated with increased risk for food insecurity. More robust measures of food insecurity are needed to help identify root causes and address the social determinants of health. By moving upstream, there can potentially be larger public health gains by getting to the heart of these issues.
Public health efforts to improve dietary patterns among the general population have mainly focused on individuallevel health changes through traditional efficacy interventions (e.g., randomized controlled trials). Such approaches have been found to be minimally effective, costly, and difficult to sustain. In order to increase the public health impact of interventions aimed at improving dietary quality, it is important to consider other designs (e.g., natural experiments) and upstream approaches. Effectiveness interventions use real world settings and can operate at multiple levels through a social ecological model. Further, they allow the ability to incorporate policy, systems, and environmental (PSE) approaches for dietary behavior changes. PSE strategies for healthy eating show promise for having higher impact with greater population reach, and they tend to be more sustainable when compared to individual behavior change approaches (Bowen et al., 2015).

Potentially impactful PSE strategies for healthy eating are built upon multiple levels and acknowledge the interrelated, dynamic, and adaptive factors that can influence dietary patterns. Some examples of PSE strategies to promote healthy eating include:

- Policy: sugary beverage taxes;

- Environment: point-of-sale incentives for fruits and vegetables, such as SNAP incentives or produce prescription programs; and

- Systems: screening for food insecurity in health care settings.

In order for healthy eating PSE strategies to have the greatest population health impact, dynamic and engaging nutrition education efforts (aimed at the "micro" individual level) combined with PSE approaches (aimed at the "macro" environment and policy levels) will be more effective than either strategy in isolation (Story \& Duffy, 2018).

Healthy eating interventions have oftentimes been developed and disseminated in a prepackaged fashion and as a one-size-fits-all approach, which may not be conducive for sustainability nor culturally tailored to racial/ethnic populations. Community engaged and community partnered research suggests that interventions can achieve better health outcomes by promoting community voice and ownership along the research continuum process (Minkler $\&$ Wallerstein, 2011). In addition, community engaged 
research can facilitate recruitment of disadvantaged and often understudied populations, narrow disparities, ensure relevance to community context, and ultimately enable communities to independently sustain intervention effects beyond limited research activities. Healthy eating interventions using PSE approaches can occur across a continuum of engagement with the community, from partnership and participation of community members throughout all stages of research, to community engagement on a smaller scale.

One strategy to engage communities in efforts to increase healthy eating may be for researchers to work more closely with the community and other public health practitioners to provide a menu of options for PSE interventions. This strategy may foster buy-in from community stakeholders and increase the uptake and sustainability of interventions. For example, a community may wish to address other health behaviors beyond healthy eating, and it is important to allow the community to drive the decisions made, forging trust and leveling the playing field between community members and researchers. Further, authentic community engagement will allow for terminology to be tailored to local cultural contexts. For instance, terminology inherently contains stigma when the term "obesity" is used, whereas using terminology that promotes positive health behaviors (e.g., healthy eating and active living) may be more appealing to communities looking to develop and implement programs in this area.

Harkening back to the need to shift toward effectiveness trials, it is frequently cited that it takes an average of 17 years for research evidence to reach clinical practice (Balas \& Boren, 2000) - or in this case, to reach community implementation. Closing the gap between research and practice for PSE strategies for healthy eating is especially challenging since the goal is not to change a single isolated factor, but to modify a string of processes that involve multiple programs and stakeholders. Understanding what works in the conditions of real world practice is a critical need. Several strategies can be employed, including:

- developing research questions in partnership with the community;

- creating collaborations in which public agencies, research partners, local organizations, and residents learn and work together to address common issues;

- employing research methods that consider the complexity of dietary patterns (e.g., pragmatic trials, time-series, natural experiments, emergent design methods);

- identifying ways to augment local data-collection systems and integrate data sharing across partners;

- emphasizing dissemination and implementation of proven healthy eating interventions and PSE strategies rather than focusing solely on new discoveries; and

- $\quad$ measuring and reporting on process of interventions with multiple levels of indicators in order to allow others to replicate studies (Kuo et al., 2015).

In order to achieve health equity, authentic community engagement needs to happen, meaning that researchers don't just pay lip service to working with communities to help change dietary patterns. Applying a health equity lens to dietary quality and using out-of-the-box methodologies are critical considerations to ultimately foster and sustain healthy eating across various populations. Community engaged approaches to address PSE factors related to diet may be an avenue to leverage local knowledge and address contextual factors that may otherwise be overlooked in traditional research studies. Generating sustainable change in communities with buy-in from multiple stakeholders is a necessary tactic, especially in light of the COVID-19 pandemic and the health and economic fallout. By conducting pragmatic trials with an eye toward speeding translation into practice and dissemination, we may finally be able to reverse chronic disease, food insecurity, obesity, and disparity trends.

Corresponding author:

Amy L. Yaroch, Ph.D.

Executive Director

Gretchen Swanson Center for Nutrition

8401 W. Dodge Rd. Suite 100

Omaha, NE, 68114

402-559-5500

ayaroch@centerfornutrition.org

iD

https://orcid.org/0000-0001-9390-2449

Courtney A. Parks

https://orcid.org/0000-0002-8989-667X

Creative Commons License:

This work is licensed under a Creative Commons Attribution-Noncommercial 4.0 International License (CC BY-NC 4.0). 


\section{References}

Balas, E. A., \& Boren, S. A. (2000). Managing clinical knowledge for health care improvement. In J. Bemmel, \& A. T. McCray (Eds.), Yearbook of medical informatics 2000: Patient-centered systems (pp. 65-70). Schattauer Verlagsgesellschaf.

Bowen, D. J., Barrington, W. E., \& Beresford, S. A. (2015). Identifying the effects of environmental and policy change interventions on healthy eating. Annual Review of Public Health, 36:289-306. https://doi.org/10.1146/annurev-publhealth$\underline{032013-182516}$

Centers for Disease Control and Prevention. (n.d.) Obesity, Race/Ethnicity, and COVID-19 . U.S. Department of Health and Human Services. Accessed October 29, 2020. https://www.cdc.gov/obesity/data/obesity-and-covid-19.html

Kuo T., Gase, L. N., \& Inkelas, M. (2015). Dissemination, implementation, and improvement science research in population health: Opportunities for public health and CTSAs. Clinical and Translational Science, 8(6):807-813. https://doi.org/10.1111/cts.12313

Minkler, M., \& Wallerstein, N. (2011). Community-Based Participatory Research for Health: From Process to Outcomes. John Wiley \& Sons.

Schanzenbach, D., \& Pitts A. (2020). Estimates of food insecurity during the COVID-19 crisis: Results from the COVID impact survey, week 1 (April 20-26, 2020). Northwestern University Institute for Policy Research. https://www.ipr.northwestern.edu/news/2020/food-insecurity-triples-for-families-during-covid.htm

Shanks, C. B., Hingle, M.D., Parks, C.A., \& Yaroch, A.L. (2020). The COVID-19 pandemic: A watershed moment to strengthen food security across the US food system. American Journal of Public Health.110(8):1133-1134.

Story, M. T., \& Duffy, E. (2018). Supporting healthy eating: Synergistic effects of nutrition education paired with policy, systems, and environmental changes. In: Nutrition education: Strategies for improving nutrition and healthy eating in individuals and communities, Vol 92. Nestlé Nutrition Institute. https://www.nestlenutrition-institute.org/docs/defaultsource/global-dcoument-library/publications/secured/nniw94-booklet---v2.pdf?sfvrsn=b2b2ced_2

U.S. Department of Health and Human Services and U.S. Department of Agriculture. (2015). 2015-2020 Dietary Guidelines. 8th edition. https://health.gov/our-work/food-nutrition/2015-2020-dietary-guidelines/guidelines/chapter-2/current-eatingpatterns-in-the-united-states/ 